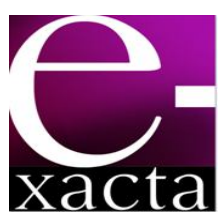

ISSN: 1984-3151

\title{
MODELAGEM DIMENSIONAL E IMPLEMENTAÇÃo DE DATA WAREHOUSE PARA GERAÇÃo DE RELATÓRIOS SOBRE Dados de TERAPIA Nutricional: Estudo dE CASO DiMENSIONAL MODELING AND IMPLEMENTATION OF DATA WAREHOUSE FOR GENERATION OF REPORTS ABOUT NUTRITION Therapy Data: CASE StUdies
}

\author{
Cleidiane Silva Ribeiro'; Silas Cesar da Silva Ribeiro² \\ Moises Henrique Ramos Pereira ${ }^{3}$ \\ 1 Bacharel em Ciência da Computação. Centro Universitário \\ de Belo Horizonte (UNIBH), 2012. Analista de Sistemas, \\ Capgemini. Belo Horizonte, MG. \\ cleidianesilvaribero@gmail.com. \\ 2 Bacharel em Ciência da Computação. Centro Universitário \\ de Belo Horizonte (UNIBH), 2012. Analista de Sistemas, \\ Resultado Real. Belo Horizonte, MG. \\ silascesar@hotmail.com. \\ 3 Mestre em Modelagem Matemática e Computacional. Cen- \\ tro Federal de Educação Tecnológica de Minas Gerais \\ (CEFET-MG), 2012. Professor Assistente no Curso de Ci- \\ ência da Computação do Uni-BH. Belo Horizonte, MG. \\ moiseshrp@gmail.com.
}

Recebido em: 13/03/2013 - Aprovado em: 12/04/2013 - Disponibilizado em: 11/06/2013

RESUMO: No Brasil, os órgãos de saúde baseiam-se nos Sistemas de Informação em Saúde (SIS) para formulação de políticas estratégicas em saúde pública. No entanto, esses SISs são geradores de grande quantidade de dados, o que tem motivado inúmeras pesquisas que visam não somente a sua integração como também à extração de conhecimento. Sendo assim, este trabalho apresenta um estudo resultante da utilização de técnicas Data Warehouse para extrair informações relevantes sobre dados de terapia nutricional com o intuito de ser utilizado como instrumento de planejamento e tomada de decisão necessária à equipe de Terapia Nutricional.

PalAVRAS-CHAVE: Data Warehouse. Terapia Nutricional. Modelagem Dimensional.

ABSTRACT: In Brazil, health agencies are based on Health Information Systems (SIS) to formulate strategic policies in public health. However, these generators are SISs large amount of data, which has motivated numerous studies aimed at not only their integration as well as knowledge extraction. Therefore, this work presents a study resulting from the use of Data Warehouse techniques to extract relevant information about nutritional therapy data in order to be used as a tool for planning and decision making necessary for Nutritional Therapy team.

KEYWORDS: Data Warehouse. Nutritional Therapy. Dimensional Modeling.

\section{INTRODUÇÃO}

O volume de informações armazenadas em bases de dados tem se tornado a grande motivação para pesquisa na área de descoberta do conhecimento em bases de dados (knowledge Discovery in Databases KDD) e Data Warehouse.

Os dados de importância para a análise de situação de saúde são inúmeros e de fontes diversas que são gerados a partir de dados sobre a população, serviços 
de saúde, mortalidade, eventos vitais, dentre outros que precisam ser organizados para facilitar o planejamento e tomada de decisão na área da saúde (SANTOS, 2011).

No Brasil existem inúmeros Sistemas de Informação em Saúde (SIS) voltados às informações hospitalares, ambulatórias, às estatísticas vitais e à investigação e controle de diversas doenças. No entanto, há certa dificuldade de interpretar os dados por eles apontados, embora as respectivas bases de dados cubram a maior parte das informações referentes à avaliação e ao planejamento de ações e serviços de saúde.

Nesse cenário, técnicas de Data Warehouse apresentam-se como uma possibilidade de representação do conhecimento, para auxiliar o processo de planejamento e tomada de decisão na área da saúde.

Date (2004) define Data Warehouse como sendo um depósito de dados orientados por assunto, integrado, variável com o tempo, para apoiar as decisões da gerência.

Algumas iniciativas de sucesso, como as apresentadas na segunda seção deste artigo, que foram obtidas através de soluções de Data Warehouse, incentivam a adoção dessa tecnologia como um agente facilitador no aumento de qualidade na gestão da saúde.

Em função disso, vários pesquisadores têm procurado aplicar técnicas de Data Warehouse para mensurar resultados desejáveis em saúde, para o planejamento e o acompanhamento das ações e dos serviços envolvidos.

O presente trabalho tem como objetivo geral aplicar técnicas de Data Warehouse em base de dados da saúde, viabilizando, dessa forma, uma reflexão e uma análise crítica sobre a utilização do KDD e Data Warehouse, respondendo às necessidades de formulação de políticas e planejamento que se constituem como uma exigência para o papel desempenhado por gestores de saúde.

Dentre os objetivos específicos, e considerando como o problema principal de pesquisa a aplicação de técnicas de Data Warehouse na área da saúde, temse:

1. Modelagem de um banco de dados relacional;

2. Implementação do banco de dados relacional;

3. Carga de dados para o banco de dados relacional;

4. Modelagem de banco de dados dimensional;

5. Implementação do banco de dados dimensional;

6. Carga de dados para o banco de dados dimensional;

7. Quantificação e qualificação dos dados;

8. Exibição dos resultados para o usuário final de maneira clara na forma de relatórios.

Nas seções subsequentes deste documento são apresentados alguns pontos relacionados à aplicabilidade de técnicas de Data Warehouse na área da saúde, com o objetivo de ressaltar as potencialidades dessa metodologia para extração do conhecimento. Será apresentada a fundamentação teórica para melhor entendimento dos termos adotados nesta pesquisa. Também será apresentada a metodologia adotada durante a realização deste trabalho, bem como os resultados obtidos $\mathrm{e}$ considerações finais.

\section{USO DE TÉCNICAS DE DATA WAREHOUSE NA ÁREA DA SAÚDE}

Devido ao exponencial aumento da quantidade de dados a serem gerenciados na área da saúde, faz-se necessário um posicionamento estratégico dos órgãos 
da área de saúde para o gerenciamento das de Data Warehouse apresentam-se como sendo uma potencial metodologia para $\circ$ gerenciamento de informações, tomada de decisão e controle de processos.

A partir da revisão da literatura, observam-se casos de aplicação de técnicas de Data Warehouse na área da saúde, como apresenta Almeida et al. (2006) em sua dissertação, que teve como objetivo principal a implementação de um projeto de Data Warehouse desenvolvido na Secretaria de Estado de Saúde de São Paulo, visando disponibilizar informação gerencial obtida através da integração de dados provenientes de diferentes sistemas de informação da saúde pública. O trabalho evidencia a grande importância da utilização de um Data Warehouse na área da saúde e detalha uma abordagem metodológica, um fluxo de atividades, uma estrutura organizacional e um conjunto de ferramentas que podem ser utilizadas eficientemente em outros projetos de Data Warehouse para a Saúde Pública.

Both, Dill e Padoin (2005) propõem em seu trabalho a utilização de Data Warehouse para solucionar as dificuldades dos gestores municipais de saúde em trabalhar com informações gerenciais estratégicas para o processo de tomada de decisão, utilizando a vasta quantidade de informações digitadas nos mais diversos sistemas de saúde disponibilizados pelo Datasus.

Neste trabalho, serão investigadas, quantificadas e qualificadas informações, em um período de três anos, dos fatores que limitam a administração plena dos processos de terapia nutricional. Para tanto, serão utilizadas técnicas de Data Warehouse.

\section{Terapia NUtRICIONAL}

A terapia nutricional pode ser classificada como informações (PINOCHET, 2011). Para tanto, técnicas terapia nutricional enteral, terapia nutricional parenteral ou terapia nutricional oral. Todas as terapias podem ser manipuladas - dieta feita com ingredientes domésticos, que deve ser liquidificada e coada - ou industrializadas - dieta pronta que é vendida comercialmente nas versões em pó, a ser diluída em água, e líquida, pronta para uso (BENTO; GARCIA; JORDÃO JUNIOR, 2011).

Nos países desenvolvidos, é comum o uso dessas dietas industrializadas. Já no Brasil, o consumo desse tipo de dieta vem aumentando gradativamente. As dietas industrializadas são práticas, nutricionalmente completas e oferecem maior segurança quanto ao controle microbiológico e composição centesimal. No entanto, não estão acessíveis para a maioria da população brasileira. A estimativa é de que cerca de $50 \%$ dos hospitais brasileiros não trabalhem com dietas industrializadas e, possivelmente, outros 20\% adquiram-nas em casos selecionados, não fazendo parte da rotina do hospital. Uma avaliação preliminar permite estimar que $30 \%$ dos hospitais trabalham com dietas industrializadas e $70 \%$ não. Embora fórmulas industrializadas e prontas para uso estejam disponíveis há mais de 20 anos, muitas instituições preferem usar as dietas preparadas na unidade hospitalar, por razões econômicas e culturais (ARAÚJO; MENEZES, 2006).

A terapia nutricional enteral é indicada para pacientes impossibilitados, por algum motivo clínico, de ingerir alimentos pela via oral ou para pacientes com baixa ingestão via oral e anorexia de diversas etiologias. A administração de nutrição pela sonda enteral não contra-indica a alimentação oral, se esta não implicar riscos para o paciente (BRITO; DREYER, 2003).

Sendo assim, a nutrição enteral consiste na infusão de uma dieta líquida administrada por meio de uma sonda colocada no estômago ou no intestino. A 
Agência Nacional de Vigilância Sanitária - ANVISA define nutrição enteral como sendo:

alimento para fins especiais, com ingestão controlada de nutrientes, na forma isolada ou combinada, de composição definida ou estimada, especialmente formulada e elaborada para uso por sondas ou via oral, industrializado ou não, utilizado exclusiva ou parcialmente para substituir ou complementar a alimentação oral em pacientes desnutridos ou não, conforme suas necessidades nutricionais, em regime hospitalar, ambulatorial ou domiciliar, visando à síntese ou manutenção dos tecidos, órgãos ou sistemas (BRASIL, 1999).

A terapia nutricional enteral é considerada parte importante do tratamento de pacientes graves em terapia intensiva, em razão do quadro de intenso catabolismo. Entretanto, vários fatores, como refluxo, diarreia e vômitos, limitam a administração plena da terapia a esses pacientes.

A terapia nutricional parenteral é indicada quando o trato gastrointestinal não pode ser utilizado ou quando não está tolerando todo o aporte calórico-protéico por via oral ou por sonda enteral, podendo-se, então, lançar mão da terapia nutricional mista (BRITO; DREYER, 2003).

A nutrição parenteral é necessária nos casos em que a alimentação oral normal não é possível ou indesejável, quando a absorção de nutrientes é incompleta e, principalmente, quando as condições mencionadas estão associadas ou podem evoluir para um estado de desnutrição. A ANVISA define nutrição parenteral como sendo:

solução ou emulsão, composta basicamente de carboidratos, aminoácidos, lipídios, vitaminas, estéril e apirogênica, acondicionada em recipiente de vidro ou plástico, destinada à administração intravenosa em pacientes desnutridos ou não, em regime hospitalar, ambulatorial ou domiciliar, visando à síntese ou manutenção dos tecidos, órgãos ou sistemas (BRASIL, 1999).

Já a nutrição oral é o tipo de nutrição mais frequente. Esta é caracterizada pela ingestão de alimentos ou nutrientes pela boca. Para que seja possível recorrer a esse tipo de nutrição, é essencial que 0 paciente mantenha a capacidade de ingestão dos alimentos
(ARAÚJO; MENEZES, 2006).

\section{Fundamentos e Métodos de Modelagem DE DATA WAREHOUSE}

Devido ao cenário competitivo do mercado, as empresas devem estar preparadas para lidar com uma grande massa de informações. É através da informação que é possível conhecer o negócio da empresa e tomar decisões. Para garantir o sucesso competitivo, as decisões devem ser tomadas cada vez mais rápido, e devem ser mais precisas, sendo quase inaceitáveis erros dos tomadores de decisões, pois pequenos deslizes podem acarretar grandes prejuízos para as organizações.

Para apoiar na tomada de decisão, surge o conceito de Data Warehouse (DW). Segundo Barbiere (2001), Data Warehouse, cuja tradução literal é Armazém de Dados, pode ser definido como um banco de dados, destinado a sistemas de apoio à decisão e cujos dados foram armazenados em estruturas lógicas dimensionais, possibilitando o seu processamento analítico por ferramentas especiais, como OLAP e Mining.

A ideia do DW é armazenar os dados espalhados pelos sistemas de uma organização, em vários graus de relacionamento e sumariação, que deverão estar processados, transformados, integrados e disponíveis, permitindo diversas formas de consultas, através dos mecanismos das ferramentas de usuários (BARBIERE, 2001).

Um grande fator contribuinte para o sucesso de um DW é o um completo entendimento do ambiente e do negócio da organização para extrair as informações relevantes que auxiliarão na tomada de decisão. Para isso é necessário o comprometimento do projetista e da organização (BOTH; PADOIN; DILL, 2005).

Segundo Barbiere (2001), os principais passos para 
implementação de um Data Warehouse são:

1. Planejamento: Nessa primeira etapa do DW, objetiva-se definir o escopo do projeto, atentando-se para as áreas críticas da empresa e para as necessidades mais prementes de informações gerencias. É feito um planejamento para integração em que são definidas as áreas e assuntos do projeto. Parte-se para estabelecer uma visão que permita amarrá-los, na medida em que as implementações forem realizadas separadamente. É nesta fase também que deve-se definir os componentes, ferramentas e sistemas básicos do DW;

2. Levantamento das Necessidades: Nessa etapa, deve-se identificar 0 modelo Dimensional ou aquele que representa os blocos conceituais de dados necessários ao alcance dos objetivos do sistema de suporte, a decisão e o modelo Fonte dos Dados. Nele são registrados os blocos conceituais de dados existentes, com suas respectivas descrições e formas atuais de armazenamento e de uso nos sistemas;

3. Modelagem Dimensional: Nesse momento o dado é consolidado e/ou sumariado nas dimensões específicas, facilitando a eficácia e rapidez das consultas mais importantes para a organização, criando-se o modelo dimensional;

4. Projeto Físico dos Bancos de Dados: Nesse passo serão desenhadas as estruturas lógicas do modelo Dimensional, com a definição das tabelas Fato e Dimensão, relacionamentos, indexação, atributos de tabelas e implantação de regras;

5. Projeto de Transformação: Nessa etapa, é feito o processo chamado ETC (Extração, Transformação e Carga);
6. Desenvolvimento de Aplicações: Nessa etapa será projetado o sistema aplicativo, objeto do trabalho. Deve-se priorizar a interface Web, facilitando o acesso aos dados e evitando a necessidade de instalação de softwares. As ferramentas escolhidas deverão possibilitar a definição de aplicativos com interfaces amistosas, geradores de relatórios, condições de visualização dos dados de forma diversas e a importação dos dados para planilhas ou processadores de textos;

7. Validação e Teste: Fase em que o sistema é testado considerando-se, ao máximo possível, as simulações de volume e de processamentos;

8. Treinamento: O grupo do treinamento deverá ser formado prioritariamente pelos usuários voltados para atividades de negócios, além dos gerentes das áreas envolvidas;

9. Implantação: É realizada por um rigoroso acompanhamento do uso das aplicações disponibilizadas, incentivando os usuários a apresentarem criticas e sugestões de melhorias para as próximas versões do sistema. Em paralelo, acontecerá a construção de um dicionário de dados fundamentais para descrever os dados do modelo Fonte, suas transformações, os dados do modelo Dimensional e suas formas de acesso e disponibilidade. Esse componente é chamado de metadados.

\section{Metodologia}

Com o intuito de projetar um Data Warehouse para armazenar dados da área da saúde de maneira a auxiliar na identificação de padrões úteis, quantificação e qualificação dos dados, foram 
definidas as seguintes etapas metodológicas: i) Aquisição dos dados; ii) Modelagem Relacional e Dimensional; iii) Exibição dos dados. A FIG. 1 apresenta uma síntese da metodologia adotada nesta pesquisa que será explicada nas subseções desta seção.

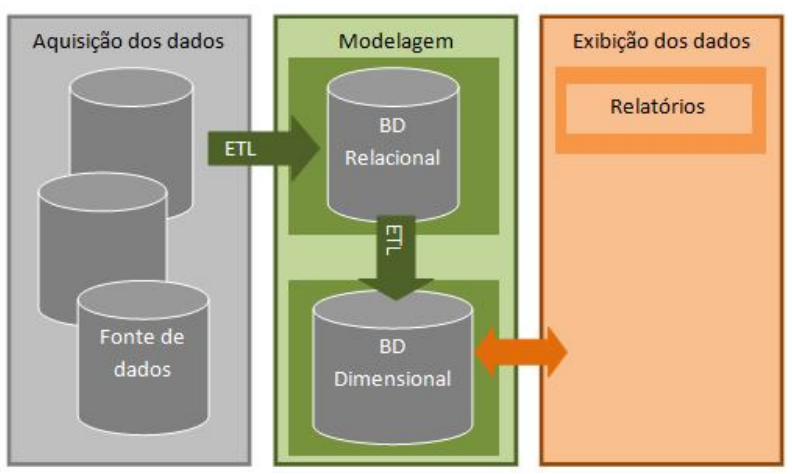

Figura 1 - Fluxo de dados do projeto

\subsection{AQUISIÇÃO DOS DADOS}

$\mathrm{Na}$ etapa de aquisição dos dados, foram utilizadas informações do Hospital Vera Cruz, casa de saúde de médio porte, situado na cidade de Belo Horizonte - MG. Os dados eram distribuídos em planilhas digitais alimentadas pelos nutricionistas do hospital.

Foram analisados dados referentes aos anos de 2009, 2011 e 2012. As bases (planilhas) são compostas de dados disponíveis no Protocolo de Terapia Nutricional (Anexo A), que são preenchidos manualmente pelos enfermeiros e posteriormente transferidos para as planilhas pelos nutricionistas.

Todo esse processo apresentado anteriormente é muito custoso, demanda muito tempo e principalmente é muito ariscado, pois as chances de se manter registros duplicados são muito grandes.

\subsection{Modelagem Relacional e Dimensional}

Nesta etapa foi desenvolvida a modelagem relacional também conhecida como Diagrama Entidade Relacionamento (DER) e a modelagem dimensional.

O desenvolvimento do modelo relacional foi necessário, pois os dados que seriam analisados encontravam-se em várias planilhas, dificultando muito o trabalho de extração de informações relevantes.

Sendo assim, foi feito todo o mapeamento das entidades e relacionamentos originando o modelo DER. A FIG. 2 apresenta o resultado da modelagem relacional do banco de dados com todas as tabelas $\mathrm{e}$ informações referentes a cada uma, de acordo com os dados do formulário do (Anexo A) e planilhas utilizadas para o levantamento dos dados no hospital.

Para carregar todos os dados, foi necessário adotar um processo de extração, transformação e carga, também conhecido como ETL. A etapa de extração baseou-se na busca das informações mais importantes em fontes externas que estejam em conformidade com a modelagem desenvolvida. Como já introduzido no subitem 5.1 desta seção, a extração dos dados se deu em planilhas digitais cedidas pelo hospital. Essa fase é muito importante, pois se os dados selecionados não forem corretamente trabalhados, as informações geradas através deles farão com que decisões sejam tomadas erroneamente. $\mathrm{Na}$ etapa de transformação foi realizada a limpeza e transformação dos dados, excluindo cadastros repetidos, padronizando abreviações e datas, excluindo atributos que podiam ser preenchidos abertamente e que não representavam o domínio do problema.

Por fim, na etapa de carga, os dados extraídos e transformados foram carregados através de imports para o banco de dados MySQL (MYSQL, 2012). Todas as etapas anteriormente citadas foram de extrema importância para que se pudesse projetar o Data Warehouse.

Sendo assim, foi realizada a modelagem dimensional, 
permitindo representar os dados em uma notação sintética, legível, objetiva, com alto desempenho. $\mathrm{Na}$
FIG. 3 pode ser visto o modelo dimensional construído, formado por tabelas Fato e por tabelas Dimensão.

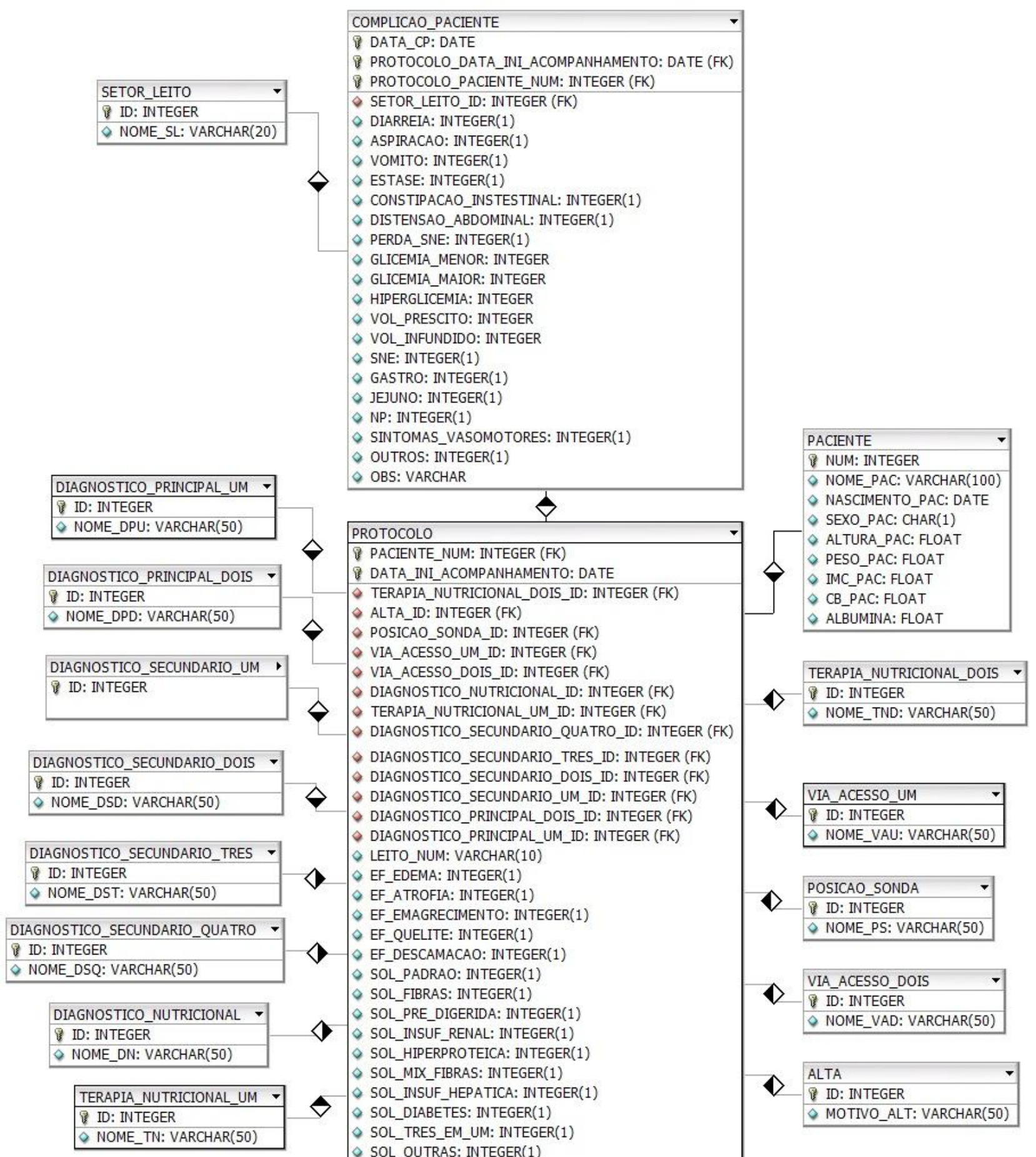

Figura 2 - Modelagem Relacional 


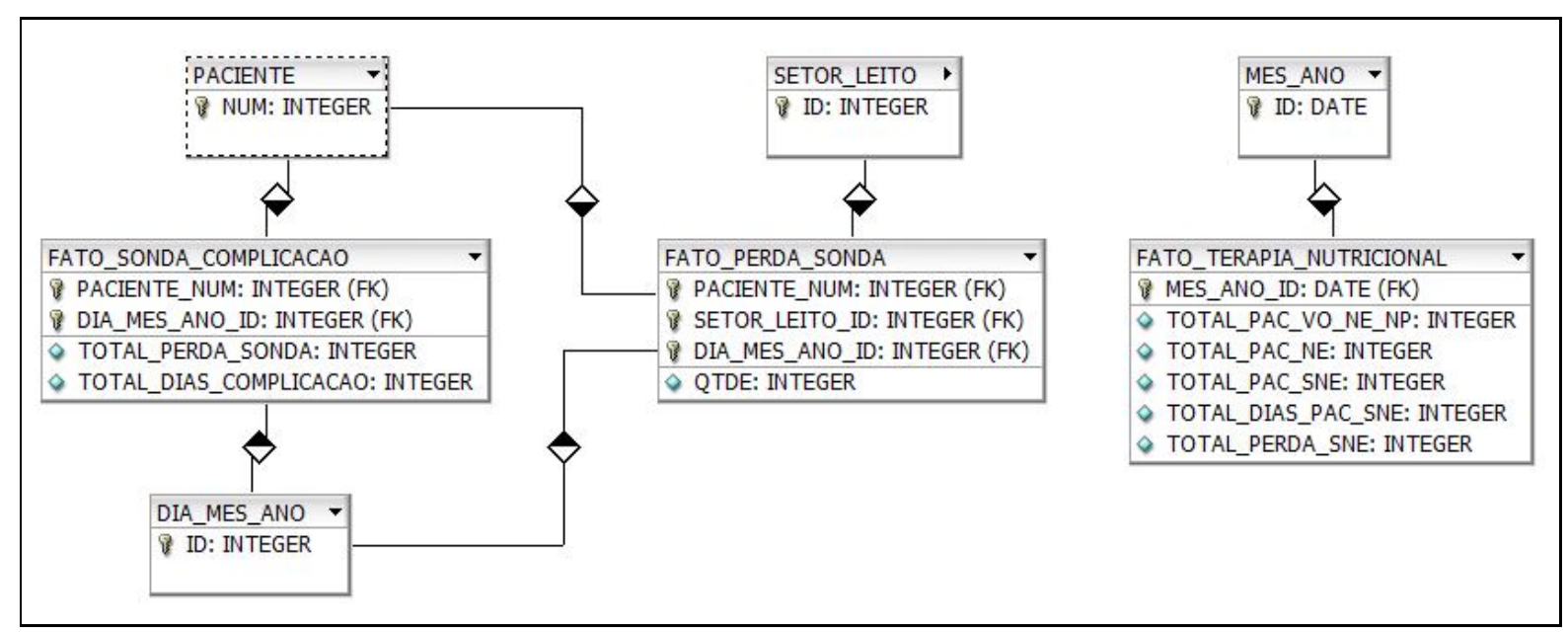

Figura 3- Modelagem Dimensional

As tabelas Dimensão são aqui representadas por: paciente, setor_leito, dia_mes_ano e mes_ano, significando entidades de negócio.

As tabelas Fato, aqui representadas por fato_perda_sonda, fato_sonda_complicacao e fato_terapia_nutricional, são formadas pelas chavesprimárias das dimensões que com elas se relacionam e armazenam valores numéricos associados a eventos do negócio. A escolha dos assuntos da composição da tabela Fato teve como escopo produzir informações a respeito dos assuntos que mais interessam aos nutricionistas do hospital.

A carga de dados do banco de dados relacional para - Data Warehouse se deu através de regras implementadas em procedures PL/SQL. Para a carga do Data Warehouse deste trabalho os dados dos anos de 2009, 2011 e 2012 foram carregados sequencialmente, mas, como a necessidade de carga é mensal, deverá ser programada no SGBD.

\subsection{EXIBIÇÃO DOS DADOS}

Esta é a etapa que mais interessa ao usuário final, pois com a exibição dos dados é possível gerenciar o conhecimento de maneira mais eficaz.

Para que esta etapa se concretizasse, foi utilizada a ferramenta Pentaho (PENTAHO, 2012), que permite fácil manipulação dos dados, geração de relatórios nos principais formatos populares: PDF, XLS, HTML e TXT. Ainda admite uma visão intuitiva, analítica e gráfica das informações de negócios e também a criação de consultas pré-definidas.

\section{Resultados}

Conforme mencionado na seção anterior, esta seção apresentará a forma como os dados são exibidos para o usuário final com uma síntese dos principais resultados obtidos através das etapas do processo de extração do conhecimento. 
As figuras 4, 5 e 6 são relatórios que apresentam de forma sintética e quantificada as principais informações utilizadas pelos nutricionistas do hospital e instâncias decisórias.

\section{Relatório mensal-Perda Sonda-Fevereiro/2012}

\begin{tabular}{|c|c|c|c|c|c|c|}
\hline Dis & Mes & Ano & Codloo do Paciente & Nome do Paciente & Selor Lelto & atole \\
\hline 21 & 2 & 2012 & 3355641 & RAIMUNDA APARECIDA GUALBERTO & Ul & 1 \\
\hline 13 & 2 & 2012 & 3355641 & RAIMUNDA APARECIDA GUALBERTO & UTI & 1 \\
\hline 14 & 2 & 2012 & 3363872 & JOEL JOAO SILVESTRE & UTI & 1 \\
\hline 28 & 2 & 2012 & 3372336 & FERNANDO ANTONIO PIZANI & UI & 1 \\
\hline 23 & 2 & 2012 & 3372336 & FERNANDO ANTONIO PIZANI & UTI & 1 \\
\hline 14 & 2 & 2012 & 3381831 & $\begin{array}{l}\text { MARIA DE LOUDES FERNANDES DA } \\
\text { SILVA }\end{array}$ & UTI & 1 \\
\hline 24 & 2 & 2012 & 3383148 & SIDENY ROSA DE SOUSA & UTI & 1 \\
\hline 14 & 2 & 2012 & 3383230 & UEIRAJARA DE CASTRO SILVA & UI & 1 \\
\hline 24 & 2 & 2012 & 3383243 & JUTAIR MARIA DE MELO & UTI & 1 \\
\hline 18 & 2 & 2012 & 3385102 & MARIA DE CARVALHO DIAS & UTI & 1 \\
\hline 21 & 2 & 2012 & 3388955 & JOSE GERALDO DA SILVA & UI & 1 \\
\hline
\end{tabular}

Figura 4 - Relatório Mensal - Perda Sonda

Relatório mensal - Total sondas perdidas X Total dias internado(a) - Fevereiro / 2012

\begin{tabular}{|llll|}
\hline Código & Nome & Total sondas perdidas & Total dias internado(a) \\
\hline 3372336 & FERNANDO ANTONIO PIZANI & 2 & 27 \\
\hline 3363872 & JOEL JOAO SILVESTRE & 1 & 17 \\
\hline 3388955 & JOSE GERALDO DA SILVA & 1 & 9 \\
\hline 3383243 & JUTAIR MARIA DE MELO & 1 & 12 \\
\hline 3385102 & MARIA DE CARVALHO DIAS & 1 & 12 \\
\hline 3381831 & MARIA DE LOUDES FERNANDES DA SILVA & 1 & 20 \\
\hline 3355641 & RAIMUNDA APARECIDA GUALBERTO & 2 & 22 \\
\hline 3383148 & SIDENY ROSA DE SOUSA & 1 & 17 \\
\hline 3383230 & UBIRAJARA DE CASTRO SILVA & 1 & 16 \\
\hline \hline
\end{tabular}

Figura 5 - Relatório mensal - Total sondas perdidas X Total dias internados(as) 
Relatório mensal-Terapia Nutricional - Fevereiro/ 2012

\begin{tabular}{|c|c|c|c|c|c|c|}
\hline Mês & Ano & $\begin{array}{l}\text { Total em dias de } \\
\text { pacientes com SNE }\end{array}$ & $\begin{array}{l}\text { Total de pacientes com } \\
\text { NE }\end{array}$ & $\begin{array}{l}\text { Total de pacientes } \\
\text { com SNE }\end{array}$ & $\begin{array}{l}\text { Total de pacientes } \\
\text { com VO+NE+NP }\end{array}$ & $\begin{array}{l}\text { Total perda } \\
\text { SNE }\end{array}$ \\
\hline Fevereiro & 2012 & 528 & 41 & 56 & 45 & 11 \\
\hline
\end{tabular}

Figura 6 - Relatório mensal - Terapia Nutricional

No "Relatório mensal - Perda Sonda - Fevereiro / 2012", é possível saber exatamente a data, o paciente, o setor do leito e a quantidade de sondas perdidas. Segundo nutricionistas, havia a necessidade de saber em que setor de leito um determinado paciente perdia mais sondas.

O "Relatório mensal - Total sondas perdidas X Total dias internado (a) - Fevereiro / 2012" aponta os pacientes que tiveram perda de sonda, exibindo o total de sondas perdidas, bem como a quantidade de dias que o paciente permaneceu internado. Com este relatório os nutricionistas tinham o controle exato da quantidade de sondas perdidas durante a internação de um determinado paciente.

Já no "Relatório mensal - Terapia Nutricional Fevereiro / 2012", há o total em dias de pacientes que usaram sonda (SNE), o total de pacientes com tipo de terapia nutricional igual à nutrição enteral (NE), o total de pacientes com sonda, total de pacientes que tiveram VO (Nutrição Via Oral), nutrição enteral (NE) e nutrição parenteral (NP) e, por fim, o total de sondas perdidas. Os totais apresentados neste relatório servem como insumo de entrada em outros sistemas.

\section{CONCLUSÕES}

Através das reuniões feitas com os nutricionistas do Hospital, foi notável a grande dificuldade que eles tinham em extrair manualmente as informações relevantes das planilhas. Este artigo apresentou a implementação de um projeto de Data Warehouse aplicado na área da saúde, mais especificamente com os dados de terapia nutricional, para obter de forma segura e prática informações relevantes para os nutricionistas.

Uma dificuldade surgida no desenvolvimento do projeto foi a quantidade de dados inconsistentes contidos nas planilhas fornecidas, obstando o processo de ETL. Foi necessário realizar seguramente a limpeza e a transformação dos dados, excluindo cadastros repetidos, padronizando abreviações e datas, eliminando atributos que podiam ser preenchidos abertamente e que não representavam o domínio do problema.

Foi perceptível que desenvolver um projeto de Data Warehouse é um processo complexo e caro, pois carece de um completo entendimento do ambiente e do negócio para extrair as informações relevantes que auxiliarão na tomada de decisão. Para isso é necessário o comprometimento do projetista e da organização.

Apesar das dificuldades encontradas, o trabalho atendeu todos os objetivos propostos e retornou, de forma rápida e segura, os relatórios com as informações relevantes desejadas para os nutricionistas do hospital.

Para trabalhos futuros fica a sugestão de implementar um sistema para os nutricionistas do Hospital Vera Cruz, para dar assiduidade com a alimentação do banco de dados de maneira 
automatizada, isentando o cadastro manual em planilhas. Além disso, poderia também aplicar técnicas de Data Mining, com o propósito de encontrar padrões nos dados e identificar informações que contribuiriam para a produção e eficácia no processo do tratamento da Terapia Nutricional no Hospital Vera Cruz.

\section{AGRADECIMENTOS}

Os autores agradecem primeiramente a Deus pela força, saúde e sabedoria para conduzir este trabalho. Agradecem aos professores Gilberto Barbosa e Diva Rodrigues, pelos conselhos: foram de muita importância. Agradecem profundamente a Tâmara Oliveira dos Reis, pelo gentil acolhimento no hospital Vera Cruz e apoio durante toda pesquisa.

\section{REFERÊNCIAS}

ALMEIDA, A. L et al. Data Warehouse para a Saúde Pública: Estudo de Caso SES-SP. 2006. Disponível em:

<http://www.sciencedirect.com/science/article/pii/S09 57417405001892>. Acesso em: 7 out. 2012.

ARAÚJO, E. M.; MENEZES, H. C. Formulações com Alimentos Convencionais para Nutrição Enteral ou Oral. 2006. Disponível em: <http://www.scielo.br/pdf/cta/v26n3/31752.pdf>.

Acesso em: 17 jun. 2012.

BARBIERE, C. BI - Business Intelligence: Modelagem e Tecnologia. Rio de Janeiro: Axcel books do Brasil, 2001. 424p.

BENTO, A. P. L; GARCIA, R. W; JORDÃO JUNIOR, A. A. Manual do Paciente em Terapia Nutricional Enteral Domiciliar. Equipe do CGPAN, 2011, 51p.

BOTH, E. L; DILL, S. L; PADOIN, E. L. Um Sistema de Apoio a Decisão para SMS. 2005. Disponível em: <http://www.sbis.org.br/cbis11/arquivos/703.pdf>. Acesso em: 8 out. 2012.

BRASIL. Agência Nacional de Vigilância Sanitária. Portaria $n^{\circ}$ 337/MS, de 14 de abril de 1999. Disponível em: <http://www.anvisa.gov.br>. Acesso em: 10 mai. 2012.
BRITO, S.; DREYER, E. Terapia Nutricional: Condutas do Nutricionista. 2003. Disponível em: $<$ http://www.hc.unicamp.br/servicos/emtn/manual_nut ricionista_2004-11-02.pdf>. Acesso em: 17 jun. 2012.

DATE, C. J. Introdução a Sistemas de Banco de Dados. 2. ed. Rio de Janeiro: Campus, 2004. 870 p. MYSQL. Disponível em: <http://www.mysql.com/>. Acesso em: 09 jun. 2012.

PENTAHO. Disponível em: <http://www.pentaho.com/>. Acesso em: 01 ago.2012

PINOCHET, L. H. C. Tendências da Tecnologia da Informação na Gestão da Saúde. 2011. 13 p. Artigo. Disponível em: <http://bases.bireme.br/cgibin/wxislind.exe/iah/online/?IsisScript=iah/iah.xis\&src =google\&base $=$ LILACS\&lang=p\&nextAction=Ink\&exp rSearch=619133\&indexSearch=ID>. Acesso em: 15 abr. 2012.

SANTOS, R. Sistema Único de Saúde. 1. Ed. Brasília: CONASS, 2011. 224 p. (Coleção Para Entender a Gestão do SUS). Disponível em: <www.conass.org.br>. Acesso em: 10 mar. 2012. 


\section{ANEXO A - Protocolo de Terapia Nutricional}

EQUIPE DE TERAPIA NUTRICIONAL E GASTROENTEROLOGIA

\section{PROTOCOLO}

Nome:

PAC:

Idade: Nascimento

Sexo:

Leito:

Data

Diagnóstico Principal:

\section{Doenças Associadas:}

\section{Avaliação Nutricional:}

Período de ingesta oral inadequada: dias

$\begin{array}{lll}\text { Exame Físico: ( ) Edema } & \text { ( )Emagrecimento } \\ & \text { ( ) Atrofia de papilas linguais } \quad \text { ( ) Quelite angular }\end{array}$

( )Descamação Cutânea

Outras alterações significativas do exame nutricional:

Peso usual:

Kg Perda de peso: $\%$

PI______ $\mathrm{Kg}$

Altura:_ $\quad \mathrm{m}$ ( 1 ) 1= medida; 2= estimada; $3=$ informada

Peso atual:

Kg ( ) 1= medida; 2= estimada; 3= informada

IMC: $\mathrm{Kg} / \mathrm{m}^{2}$

$\mathrm{CB}$ : $\mathrm{cm}$

PCT: $\mathrm{mm}$

Diagnóstico Nutricional:

Cálculo das necessidades calóricas iniciais:

GEB: Kcal GET: kcal FA:

FI: método: Harris Benedict

GET: método direto

\section{Terapia Nutricinal indicada:}

\section{( ) Nutrição Enteral}

Posição da sonda: ( ) Gástrica

( ) Intestinal - ( ) às cegas ( ) EDA

Solução: ( ) Padrão

( ) Fibras Insolúveis

( ) Pré Digerida

( ) Insuf. Renal

( ) Outros

Tipo de sonda:

( ) SNE

( ) Gastrostomia endoscópica
( ) SNG

( ) Gastrostomia cirúrgica
( ) Diabetes

( ) Hiperproteica

( ) Mix de fibras

\section{( ) Nutrição Parenteral}

Posição do （）Subclávia ___ ( ) Periférico

cateter: ( ) Jugular Interna

Data da punção: / / .

$\mathrm{N}^{\mathrm{o}}$ de punção:

Lumem: ( ) Simples （ ) Duplo ( ) Triplo 
( ) jejunostomia endoscópica ( ) jejunostomia cirúrgica $\mid$ ( ) Outras

Desfecho: ( ) Alta da NUTRI-GEN （ ）Terapia Nutricional domiciliar ( ) Óbito ( ) Alta da NUTRI-GEN /piora clinica 


\section{Complicações da NE}

\begin{tabular}{|c|c|c|c|c|c|c|c|c|c|c|c|c|c|}
\hline \multirow[t]{2}{*}{ Data } & \multirow[t]{2}{*}{\begin{tabular}{|l|} 
Diar- \\
réia**
\end{tabular}} & \multirow[t]{2}{*}{ Aspiração } & \multirow[t]{2}{*}{ Vômito } & \multirow[t]{2}{*}{ Estase** } & \multirow[t]{2}{*}{$\begin{array}{l}\text { Constipação } \\
\text { Intestinal*** }\end{array}$} & \multirow[t]{2}{*}{$\begin{array}{l}\text { Distensão } \\
\text { Abdominal }\end{array}$} & \multirow[t]{2}{*}{\begin{tabular}{|c|} 
Perda de \\
S.N. E \\
\end{tabular}} & \multirow[t]{2}{*}{ Glicemia } & \multirow[t]{2}{*}{$\begin{array}{c}\text { Volume } \\
\text { Prescrito } \\
\end{array}$} & \multirow[t]{2}{*}{\begin{tabular}{|c|}
$\begin{array}{c}\text { Volume infun- } \\
\text { dido }\end{array}$ \\
\end{tabular}} & \multirow[t]{2}{*}{$\begin{array}{c}\text { Sintomas } \\
\text { vasomotores }\end{array}$} & \multirow[t]{2}{*}{ Outros } & \multirow[t]{2}{*}{ Observação } \\
\hline & & & & & & & & & & & & & \\
\hline & & & & & & & & & & & & & \\
\hline & & & & & & & & & & & & & \\
\hline & & & & & & & & & & & & & \\
\hline & & & & & & & & & & & & & \\
\hline & & & & & & & & & & & & & \\
\hline & & & & & & & & & & & & & \\
\hline & & & & & & & & & & & & & \\
\hline & & & & & & & & & & & & & \\
\hline & & & & & & & & & & & & & \\
\hline & & & & & & & & & & & & & \\
\hline & & & & & & & & & & & & & \\
\hline & & & & & & & & & & & & & \\
\hline & & & & & & & & & & & & & \\
\hline & & & & & & & & & & & & & \\
\hline & & & & & & & & & & & & & \\
\hline & & & & & & & & & & & & & \\
\hline & & & & & & & & & & & & & \\
\hline & & & & & & & & & & & & & \\
\hline & & & & & & & & & & & & & \\
\hline & & & & & & & & & & & & & \\
\hline & & & & & & & & & & & & & \\
\hline & & & & & & & & & & & & & \\
\hline & & & & & & & & & & & & & \\
\hline & & & & & & & & & & & & & \\
\hline & & & & & & & & & & & & & \\
\hline & & & & & & & & & & & & & \\
\hline & & & & & & & & & & & & & \\
\hline & & & & & & & & & & & & & \\
\hline
\end{tabular}

